Bundesarbeitsgemeinschaft kommunaler Frauenbüros und Gleichstellungsstellen (BAG) (Hg.), 2018: Antifeminismus als Demokratiegefährdung?! Gleichstellung in Zeiten von Rechtspopulismus. Berlin. Internet: www. frauenbeauftragte.org/sites/default/files/uploads/downloads/antifeminismus_als_demokratiegefaehrdung.pdf (09.01.20).

BT-Drucksache 19/8220, 2019: Antwort der Bundesregierung vom 27.03.2019 auf die Kleine Anfrage der Abgeordneten Dr. Marc Jongen, Dr. Götz Frömming, Nicole Höchst, weiterer Abgeordneter und der Fraktion der AfD: „Genderkritik und die Gefahr der Spaltung der Gesellschaft durch Misandrie", 13.03.2019. Internet: http://dipbt.bundestag.de/doc/btd/19/087/1908788.pdf (08.01.2020).

Diskursatlas Antifeminismus, o.J. Internet: www.diskursatlas.de/index.php?title=Hauptseite (10.02.20).

Henninger, Annette, 2019: Antifeminismus in Deutschland: Entwicklungen in verschiedenen Praxisfeldern. In: Femina Politica 28 (1), 139-141.

Henninger, Annette/Birsl, Ursula (Hg.), 2020 (i.E.): Antifeminismen. .Krisen'-Diskurse mit gesellschaftsspaltendem Potential? Bielefeld: transcript.

Kelle, Birgit, 2017: Hört auf, uns Mütter „,befreien“ zu wollen! In: welt.de, 13.07.2017. Internet: www.welt.de/debatte/kommentare/article166632672/Hoert-auf-uns-Muetter-befreien-zu-wollen.html (08.01.20).

Klemm, Sarah/Wittenzellner, Ulla/Knepper, Lena, o.J.: Dieses Genderdings! Wörterbuch. Berlin: Dissens-Institut für Bildung und Forschung e.V.. Internet: https://somi.dissens.de/fileadmin/ social_media_interventions/SoMI_Wörterbuch.pdf (10.02.20).

Marx, Daniela/Kotlenga, Sandra, 2017: Übliche Widerstände oder neue Infragestellungen? Gleichstellungsfeindlichkeit und Angriffe auf Gleichstellungsarbeit an Hochschulen in Niedersachsen. Bericht zum Projekt „Antifeminismus an Hochschulen in Niedersachsen“ (unter Mitarbeit von Birte Driesner und Doris Hayn). Georg-August-Universität Göttingen: Landeskonferenz Niedersächsischer Hochschulbeauftragter. Internet: https://www.nds-lagen.de/download/ Berichte/Abschlussbericht_LNHF-Projekt_Antifem_final_Sept_2018.pdf (09.01.20).

Schutzbach, Franziska, 2017: „Gender Raus!“ Zwölf Richtigstellungen zu Antifeminismus und Gender-Kritik. Hrsg. von der Heinrich-Böll-Stiftung und der Rosa-Luxemburg-Stiftung, Berlin. Internet: https://www.gwi-boell.de/de/2017/07/04/gender-raus-12-richtigstellungen-zu-antifeminismus-und-gender-kritik (09.01.20).

\title{
Frauen auf der Überholspur? Kolonialität der Staatsbürgerschaft und verkörperte soziale Mobilität ${ }^{1}$
}

\author{
JULIA ROTH. MANUELA BOATCĂ
}

Die gegenwärtige Kluft zwischen Arm und Reich auf globaler Ebene macht die Welt ungleicher als je zuvor (Reid-Henry 2015). Gleichzeitig ist der Reichtum stark geschlechtsspezifisch verteilt. Unter zehn Milliardär*innen weltweit ist nur eine Frau, und nur 1,4\% darunter sind Milliardärinnen der ersten Generation, also keine Erbinnen (Wang 2018; Pendleton/Cannon 2018). Im Gegensatz dazu besitzen Frauen weniger als zwei Prozent Land weltweit, stellen die Mehrheit der ärmsten Menschen 
der Welt dar und leisten jährlich zehn Billionen Dollar an unbezahlter Pflege- und Sorgearbeit (Oxfam 2018). An beiden Enden der Weltvermögens- und Einkommensverteilung erklären die geschlechtsspezifischen Unterschiede einen großen Teil der derzeit zunehmenden globalen wirtschaftlichen Ungleichheiten.

Da die Unterschiede bei den Durchschnittseinkommen zwischen den Ländern zusammen mit der globalen Kluft zwischen Arm und Reich immer größer werden, ist Migration zu einer der wirksamsten Strategien der Aufwärtsmobilität geworden (Korzeniewicz/Moran 2009; Milanovic 2016; Shachar 2009; Reid-Smith 2015). Der Zugang zum Territorium und zu den Ressourcen eines Landes, das relativ bessergestellt ist als das Geburts- oder Wohnsitzland, bringt den Menschen in den meisten Teilen der Welt unmittelbare wirtschaftliche Vorteile. Branko Milanovic (2016) hat den Begriff „Staatsbürgerschaftsprämie“ geprägt, um zu erklären, dass die Geburt in einem reichen Land eine Besserstellung bezüglich der Einkommensverteilung gegenüber jemandem bedeutet, der in einem sehr armen Land geboren wurde. Je nachdem, wo sie sich befinden und wohin sie einwandern können, können die Bürger*innen armer Länder also ihr Realeinkommen verdoppeln, verdreifachen oder mehr als verzehnfachen, wenn sie in ein reiches Land ziehen (ebd.).

Doch der Zugang zur internationalen Migration in ein reicheres Land ist ungleich verteilt. Auch die individuellen Möglichkeiten, Ungleichheit und Armut zu bekämpfen, sind geschlechtsspezifisch verschieden. Das Wissen um mögliche Reiserouten und bessere wirtschaftliche Aussichten, die Transportkosten (ob legal oder unautorisiert) und die Reisekosten erfordern erhebliche physische Mobilität sowie materielle und immaterielle Ressourcen. Solche Ressourcen stehen vor allem Mitgliedern der Mittel- und Oberschicht, Gebildeten und denjenigen zu, die nicht rassistisch markiert werden sowie Männern, die alleine reisen können. Als nicht-westlich rassisierte Frauen (insbesondere in Begleitung von Kindern) und andere marginalisierte Personen mit non-konformer Gender-Performance sind gegenwärtig aufgrund fortbestehender kolonialer und Genderhierarchien immer noch die am stärksten gefährdeten Migrierenden. Wir sprechen deshalb von der anhaltenden Kolonialität der Staatsbürgerschaft.

Im Folgenden argumentieren wir daher, dass, im Gegensatz zu überwiegend männlichen wohlhabenden Investoren, die ihre globale Mobilität erkaufen können, Frauen und feminisierte Andere, insbesondere LGBTIQ und rassisierte Personen, ihre vergeschlechtlichten Körper in langwierigen Arrangements zum Tausch anbieten müssen, um so schließlich Aufwärtsmobilität durch eine Aufenthaltserlaubnis oder die Staatsbürgerschaft eines wohlhabenden Landes zu erlangen. Der durch das ökonomische Kapital vermittelte Zugang von Frauen und feminisierten Anderen zu sozialer Mobilität ist also sowohl mit prekäreren Mitteln (dem eigenen Körper), als auch mit prekäreren Ergebnissen als bei Männern und unmarkierten Personen verbunden. Wir argumentieren, dass die wirtschaftliche Macht von Frauen teilweise als Widerstand funktioniert, sie also der Kolonialität der Macht entgegenwirkt, die sie systematisch in prekärere Positionen in der globalen Mobilitätsstruktur verbannt hat. 